\title{
Reparixin, an Inhibitor of CXCR1 and CXCR2 Receptor Activation, Attenuates Blood Pressure and Hypertension-Related Mediators Expression in Spontaneously Hypertensive Rats
}

\author{
Hye Young Kim, ${ }^{a}$ Jin Hee CHOI, ${ }^{a}$ Young Jin KAng, ${ }^{b}$ So Young PARK, ${ }^{c}$ Hyoung Chul CHOI, ${ }^{b}$ and \\ Hee Sun $\mathrm{KIM}^{*, a}$ \\ ${ }^{a}$ Department of Microbiology, Aging-associated Vascular Disease Research Center, College of Medicine, Yeungnam \\ University; ${ }^{b}$ Department of Pharmacology, Aging-associated Vascular Disease Research Center, College of Medicine, \\ Yeungnam University; and ${ }^{c}$ Department of Physiology, Aging-associated Vascular Disease Research Center, College of \\ Medicine, Yeungnam University; 317-1 Daemyungdong, Namgu, Daegu 705-717, South Korea. \\ Received August 5, 2010; accepted October 5, 2010; published online October 13, 2010
}

Reparixin, an inhibitor of CXCL8 receptor CXCR1 and CXCR2 activation, has been shown to attenuate inflammatory responses in various injury models. In the present study, the hypertension-related functional roles of reparixin were examined in hypertensive animals. Spontaneously hypertensive rats (SHR) at the age of 18 weeks were administered a subcutaneous injection of reparixin $(5 \mathrm{mg} / \mathrm{kg})$ daily for 3 weeks $(\mathrm{SHR}-\mathrm{R}, n=5)$. Control groups consisted of normal saline-treated SHR (SHR-N, $n=5)$ and normotensive Wistar-Kyoto rats (WKY-N, $n=5)$. Reparixin effectively decreased systolic blood pressure and increased the blood flow. The thoracic aorta wall thickness was significantly decreased in SHR-R compared to SHR-N. Expressions of CXCL8, CCL2, 12lipoxygenase (LO) and endothelin (ET)-1 were significantly decreased in SHR-R thoracic aorta tissues compared to SHR-N. Furthermore, expression of angiotensin II subtype I receptor $\left(\mathrm{AT}_{1} \mathrm{R}\right)$ protein was decreased in SHR-R thoracic aorta tissues compared to SHR-N. In addition, the plasma levels of nitric oxide were slightly elevated in SHR-R compared to the levels in SHR-N. These findings indicate that inhibition of hypertension-related mediators by reparixin results in the reduction of blood pressure in SHR. Therefore, these results suggest that reparixin-mediated blockade of CXCL8 receptor activation attenuates vascular hypertension in SHR.

Key words reparixin; hypertension; CXCR2

Reparixin $[R(-)-2-(4-i s o b u t h y l p h e n y l)$ propionyl methanesulfonamide] (formerly repertaxin) is a non-competitive allosteric blocker of CXCR1 and CXCR2 receptor activation, which inhibits intracellular signal pathways without affecting receptor bindings. ${ }^{1)}$ It has been shown to be efficacious in several injuries including acute lung injury, peritonitis, traumatic spinal cord injury and ischemia/reperfusion injury. ${ }^{1-7)}$

CXCR1 and CXCR2 are G protein coupled receptors (GPCRs) that bind glutamine-leucine-arginine-positive $($ ELR +$)$ chemokines with high affinity. Blockade of GPCRs appears to be a valid therapeutic strategy for the treatment of ischemia/reperfusion injuries. ${ }^{1,3,6,7)}$ CXCR1 and CXCR2 possess high sequence homology at the amino acid level except in their amino-terminal regions. However, CXCR1 is absent in rodents and exhibits a slightly lower affinity for CXCL8, the most widely characterized ELR+ chemokine in vitro. ${ }^{3,8)}$ Dunstan et al. identified the rat orthologs of human CXCL8 receptors and reported that rat CXCR1-like and CXCR2 are most closely related to the human CXCL8 receptor genes. ${ }^{9)}$ CXCR1 and CXCR2 can bind to other ELR+ chemokines with high affinity, but with lower affinity than CXCL8. ${ }^{7}$ Reparixin potently and selectively inhibits a wide range of biological activities that are induced by CXCL8 such as leukocytes recruitment and functional inflammatory reactions. ${ }^{10)}$ However, reparixin does not affect CXCR1/CXCR2 activation induced by other chemotactic factors, C5a, fMLP, CXCL12 or several other agonists of GPCRs. ${ }^{1,3,10-12)}$

CXCL8 regulates macrophages, endothelial cells and vascular smooth muscle cells (VSMCs) and functions via binding to CXCR1 and CXCR2 to initiate downstream signaling. Interestingly, CXCL8 has been implicated in hypertensive inflammatory changes in arterial walls..$^{10,13-15)}$ Elevated CXCL8 expression and CXCL8-induced production of 12lipoxygenase (LO) were observed in hypertensive arterial walls and VSMCs of spontaneously hypertensive rats (SHR). ${ }^{13,15)}$ Therefore, inhibition of CXCL8 receptor activation may be a useful approach to assess a range of CXCL8related hypertensive biological activities in SHR. Although reparixin is a well-known potent and specific inhibitor of CXCL8-induced activity during inflammatory neutrophil recruitment, its effect on vascular hypertension has not yet been addressed.

Therefore, in this study, the effects of reparixin on blood pressure, aortic wall thickness and expression patterns of hypertension-related mediators, including angiotensin II receptors, CCL2, CXCL8, 12-lipoxygensae (LO) and endothelin (ET)-1, in SHR were examined.

\section{MATERIALS AND METHODS}

Reagents The trizol reagent for total RNA isolation was purchased from Invitrogen (Carlsbad, CA, U.S.A.). Phosphate-buffered saline (PBS), Dulbecco's modified Eagle's medium (DMEM), penicillin-streptomycin and fetal bovine serum (FBS) were purchased from Gibco/BRL (Life Technologies, Gaithersburg, MD, U.S.A.). Recombinant human CXCL8 was purchased from R\&D systems (Minneapolis, MN, U.S.A.). Oligonucleotide primers for CCL2, CXCL8, 12-LO, ET-1, Angiotensin II (Ang II) subtype 1 receptor $\left(\mathrm{AT}_{1} \mathrm{R}\right)$, Ang II subtype 2 receptor $\left(\mathrm{AT}_{2} \mathrm{R}\right)$, and $\beta$-actin polymerase chain reaction (PCR) were synthesized by Bionics (Seoul, Korea). LightCycler FastStart DNA SYBR Green I 
Mix was purchased from Roche (Mannheim, Germany). Reparixin was purchased from Sigma-Aldrich (St. Louis, CA, U.S.A.). Mouse anti-human CXCL8 antibody was purchased from BD Biosciences (BD Pharmingen, San Diego, CA, U.S.A.). The rabbit anti-rat CCL2 antibody was purchased from Peprotech (London, U.K.). Rabbit anti-human $\mathrm{AT}_{1} \mathrm{R}$ and $\mathrm{AT}_{2} \mathrm{R}$ antibodies were purchased from Abcam (Cambridge, U.K.). Goat anti-human 12-LO and ET-1 polyclonal antibodies were purchased from Santa Cruz Biotechnology (Santa Cruz, CA, U.S.A.). All other reagents were from pure-grade commercial preparations.

Animals and Experimental Protocols Pathogen-free male inbred normotensive Wistar Kyoto rats (WKY) and SHR were purchased from Japan SLC Inc. (Shizuoka, Japan). All experimental animals received autoclaved food and were bedded to minimize exposure to viral and microbial pathogens. Rats were cared for in accordance with the Guide for the Care and Use of Experimental Animals of Yeungnam Medical Center. The study protocol was reviewed and approved by the Committee on the Ethics of Animal Experiments, College of Medicine, Yeungnam University.

The reparixin-treated group contained 5 SHR (SHR-R), where equal numbers of normal saline-treated SHR (SHR-N) and WKY (WKY-N) served as controls. Eighteen-week-old SHR received a subcutaneous injection of reparixin (5 $\mathrm{mg} / \mathrm{kg}$ ) once per day for 3 weeks. Reparixin effects on blood flow, blood pressure and body weight were measured before treatment and then weekly until 1 week after the final injection. The effect of reparixin on the expression of hypertension-related mediators in thoracic aortas, as well as nitric oxide (NO) plasma levels, was examined 1 week after the final injection. At the end of the 4 week period, both control and reparixin-treated rats were anesthetized via intraperitoneal injection of thiopental $(50 \mathrm{mg} / \mathrm{kg})$, and blood and tissue samples were collected.

Measurement of Systolic Blood Pressure and Blood Flow Systolic blood pressure was recorded using the tailcuff method (Data Acquisition system; AD Instrument Pty. Ltd., Sydney, Australia) in conscious rats. Rats were restrained inside clear Perspex rat restrainers and placed on a preheated base plate $\left(38-40^{\circ} \mathrm{C}\right)$ for $35 \mathrm{~min}$. Several systolic blood pressure readings were recorded for each rat and four median systolic blood pressure readings, in which the differences were within $10 \mathrm{mmHg}$, were averaged. The average of four median readings was used as the mean systolic blood pressure. Following the systolic blood pressure measurements, blood flow was measured in tail part of the rat using a laser tissue blood flowmeter (Laser Tissue Blood Flowmeter; Omega Wave, Tokyo, Japan).

Immunohistochemistry To measure the thickness of the thoracic aorta wall, transverse sections of $4 \%$ paraformaldehyde-fixed, paraffin-embedded thoracic aorta were placed on coated slides and stained with Masson's trichrome. The thoracic aorta wall thickness and wall : lumen ratios were calculated using the following formulas: outer diameter (OD)inner diameter (ID)/2 and (OD-ID)/ID, respectively. ${ }^{16}$ )

Aortic cross sections were stained immunohistochemically for CXCL8, CCL2, $\mathrm{AT}_{1} \mathrm{R}$ and $\mathrm{AT}_{2} \mathrm{R}$. To assess CCL2, CXCL8, $\mathrm{AT}_{1} \mathrm{R}$ and $\mathrm{AT}_{2} \mathrm{R}$ expression, slides fixed with aortic cross sections were treated with pepsin for antigen retrieval, and endogenous peroxidase was blocked with $3 \% \quad \mathrm{H}_{2} \mathrm{O}_{2}$.
After blocking with normal blocking serum for $30 \mathrm{~min}$ at room temperature (RT), sections were incubated with the anti-CXCL8 antibody $(1: 50)$, anti-CCL2 antibody (1:50), anti-AT $\mathrm{R}_{1}$ antibody $(1: 50)$ and anti-AT ${ }_{2} \mathrm{R}$ antibody $(1: 300)$ for $1 \mathrm{~h}$ at RT and horse-radish peroxidase (HRP)-conjugated rabbit anti-mouse immunoglobulin $\mathrm{G}$ (IgG) antibody for $30 \mathrm{~min}$ at RT. To visualize staining, sections were developed with the 3,3'-diaminobenzidine substrate-chromogen system for $5 \mathrm{~min}$ at RT.

VSMCs Preparation VSMCs were obtained using an explant method from the thoracic aortas of 16- to 22-weekold male SHR and WKY as described by Kim et al. ${ }^{15}$ ) VSMCs were cultured in DMEM supplemented with $10 \%$ FBS and 1\% penicillin-streptomycin. Cells were detached with $0.25 \%$ trypsin/ethylenediaminetetraacetic acid (EDTA) and seeded into $75 \mathrm{~cm}^{2}$ tissue culture flasks at a density of $10^{5}$ cells $/ \mathrm{ml}$. All experiments were conducted during cell passages 3 to 7. Prior to stimulation, 95\% confluent VSMCs were serum-starved overnight in DMEM containing $0.1 \%$ FBS. Cell cultures were incubated in a humidified incubator at $37^{\circ} \mathrm{C}$ and $5 \% \mathrm{CO}_{2}$ in the presence or absence of stimuli for the indicated time.

Human Umbilical Vein Endothelial Cells (HUVECs) Culture HUVECs were isolated from human umbilical cords collected from normal deliveries at Yeungnam University. A signed consent was obtained and the study was approved by the Institutional Review Board of Yeungnam University Medical Center. Vein in cord was washed with prewarmed phosphate buffered saline (PBS) using a $50 \mathrm{ml}$ syringe and then filled with Hanks' balanced salt solution (HBSS) containing $0.2 \%$ collagenase type II. After $15 \mathrm{~min}$, HBSS was collected into a tube and centrifuged at $2000 \mathrm{rpm}$ for $5 \mathrm{~min}$. Isolated cells were planted in $100 \mathrm{~mm}$-diameter culture dishes coated with $0.1 \%$ gelatin (Sigma-Aldrich, Oakville, ON, Canada) and cultured in M199 containing $10 \%$ fetal bovine serum and $1 \%$ antibiotics. Cells were grown at $37^{\circ} \mathrm{C}$ in a humidified atmosphere with $5 \% \mathrm{CO}_{2}$. Cells planted on the culture dish were regarded as passage 0 and the cells from passages 3-4 were used.

Preparation of Total RNA and Real-Time PCR Total RNA from thoracic aortas or VSMCs was extracted using Trizol according to the manufacturer's instructions. Total RNA was quantified by measuring the optical density (OD) at 260 and $280 \mathrm{~nm}$. CCL2, CXCL8, AT 1 R, AT 2 R, 12-LO and ET-1 cDNA was obtained from VSMCs by reverse transcription of $1 \mu \mathrm{g}$ total RNA. The cDNAs were then subjected to real-time PCR using a LightCycler. Each $20 \mu$ l reaction contained LightCycler FastStart DNA SYBR Green I mix, primer and $2 \mu \mathrm{l}$ of cDNA. Prior to PCR amplification, the mixture was incubated at $95^{\circ} \mathrm{C}$ for $10 \mathrm{~min}$. The amplification program consisted of 45 cycles of denaturation $(10 \mathrm{~s}$ at $95^{\circ} \mathrm{C}$ ), annealing $(5 \mathrm{~s}$ at the primer-appropriate temperature), and extension $\left(10 \mathrm{~s}\right.$ at $\left.72^{\circ} \mathrm{C}\right)$ with fluorescence detection at $72^{\circ} \mathrm{C}$ after each cycle. After the final cycle, analysis of the melting point of each sample was performed over the range of 65 to $95{ }^{\circ} \mathrm{C}$ with continuous fluorescence detection. $\beta$ Actin expression levels were used for normalization. The results for each gene are expressed as the expression level relative to the corresponding $\beta$-actin expression level. The primers used for PCR were as follows: CXCL8 (365 bp) sense, 5'-gaagatagattgcaccga-3', antisense, 5'-catagcctctca- 
cacatttc-3'; CCL2 (396 bp) sense, 5'-cctgttgttcacagttgctgcc$3^{\prime}$, antisense, $5^{\prime}$-tctacagaagtgcttgaggtggttg- $3^{\prime} ; \mathrm{AT}_{1} \mathrm{R}$ (445 bp) sense, 5'-cacctatgtaagatcgcttc-3', antisense, 5'-gcacaatcgccataattatcc-3'; $\mathrm{AT}_{2} \mathrm{R}(65 \mathrm{bp})$ sense, $5^{\prime}$-ccgtgaccaagtcttgaagatg-3', antisense, $5^{\prime}$-agggaagccagcaaatgatg-3'; 12-LO (312 bp) sense, 5' 'tggggcaactggaagg-3', antisense, 5'-agagcgcttcagcaccat-3'; ET-1 (370 bp) sense, 5'-ctcctccttgatggacaagg- $3^{\prime}$, antisense, $5^{\prime}$-cttgatgctgttgctcatgg- $3^{\prime} ; \beta$-actin (101 bp) sense, $5^{\prime}$-tactgccetggctcctagca- $3^{\prime}$, antisense, $5^{\prime}$-tggacagtgaggccaggatag-3'. CCL2, CXCL8, $\mathrm{AT}_{1} \mathrm{R}, \mathrm{AT}_{2} \mathrm{R}, 12-\mathrm{LO}$ and ET1 mRNA levels were determined by comparing experimental levels to standard curves. mRNA levels were expressed as the fold increase or decrease in expression.

Western Blotting Total lysates were prepared in PROPREP buffer (iNtRON, Seoul, Korea). Protein concentrations were determined by a Bradford assay (Bio-Rad, Hercules, CA, U.S.A.) using bovine serum albumin as a standard. Twenty microgram protein samples were separated on $10 \%$ sodium dodecyl sulfate (SDS)-polyacrylamide gels and then transferred to nitrocellulose membranes. The membranes were soaked in $5 \%$ nonfat dried milk in TBST $(10 \mathrm{mmol} / 1$ Tris/ $\mathrm{HCl} \mathrm{pH} \mathrm{7.5,} 150 \mathrm{mmol} \mathrm{NaCl}$ and $0.05 \%$ Tween-20) for $1 \mathrm{~h}$ and then incubated for $16-18 \mathrm{~h}$ with primary antibodies against 12 -LO, ET- 1 and $\gamma$-tubulin at $4{ }^{\circ} \mathrm{C}$. Membranes were washed three times with TBST for $10 \mathrm{~min}$ and then incubated with horseradish peroxidase-conjugated secondary antibody for $1 \mathrm{~h}$ at room temperature. The membranes were rinsed three times with TBST for $10 \mathrm{~min}$ and antigen-antibody complexes were detected using the enhanced chemiluminescence detection system (LAS-3000; Fujifilm, Tokyo, Japan).

Determination of Plasma Level of Nitric Oxide (NO) Quantitative measurement of NO production was performed using a nitrate/nitrite colorimetric assay kit (Cayman Inc., Ann Arbor, Michigan, U.S.A.) according to the manufacturer's instructions. Blood samples were centrifuged at $2500 \mathrm{rpm}$ for $20 \mathrm{~min}$ and isolated plasma was then transferred to a 96 well plate in $40 \mu \mathrm{l}$ aliquots. Ten microliters of enzyme cofactor mixture and $10 \mu \mathrm{l}$ of nitrate reductase mixture were added to each well, and the plate was covered and incubated at room temperature for $3 \mathrm{~h}$. Next, $100 \mu \mathrm{l}$ of Greiss reagent $(0.5 \%$ sulfanilamide, $2.5 \%$ orthophosphoric acid, and $0.25 \%$ naphthylenediamine) were added to the wells for $10 \mathrm{~min}$ and the absorbance was read at $540 \mathrm{~nm}$ using a microplate reader (SUNRISE, TECAN, Sydney, Australia)

Statistical Analysis Results were expressed as mean \pm S.E.M. from repeated experiments when comparing between groups. Statistical significance was determined by the Mann-Whitney test using SPSS v. 17.0. Differences between data sets for systolic blood pressure and blood flow were assessed by analysis of variance (ANOVA) followed by Bonferoni's $t$-test.

\section{RESULTS}

Reparixin Treatment Decreases Systolic Blood Pressure and Increases Blood Flow in SHR Reparixin treatment did not significantly influence body weight in SHR, while the rats showed an age-related increase in body weight (18 weeks of age: $355.6 \pm 7.3 \mathrm{~g}, 22$ weeks of age: $374.38 \pm$ $13.2 \mathrm{~g}$ ). Reparixin-treated SHR (SHR-R) showed a reduction in systolic blood pressure (SHR-R: 185 \pm 4 , SHR-N: 212 \pm 2 ,
WKY-N: $139 \pm 6 \mathrm{mmHg}, p<0.01)$, accompanied by an increase in blood flow (SHR-R: $6.2 \pm 0.2$, SHR-N: $4.7 \pm 0.4$, WKY-N: $7.0 \pm 0.3 \mathrm{ml} / \mathrm{min} / \mathrm{g}, p<0.01$ ) (Fig. 1).

Reparixin Decreases Thoracic Aorta Wall Thickness in SHR Representative thoracic aorta walls from WKY-N, SHR-N and SHR-R are shown in Fig. 2A. SHR-R rats exhibited a significant reduction in vascular wall thickness compared to SHR-N and the SHR-R vascular walls closely resembled those in WKY-N. The aortic wall thickness for each group was as follows; SHR-R: 106 \pm 1.79 , SHR-N: $116 \pm 1.79$, WKY-N: $101 \pm 1.82 \mu \mathrm{m}$. In addition, the wall to lumen ratio of thoracic aorta for each group was as follows; SHR-R: $0.13 \pm 0.01$, SHR-N: $0.16 \pm 0.005$, WKY-N: $0.13 \pm$ 0.005) (Fig. 2B).

Reparixin Decreases Chemokines and $\mathrm{AT}_{1} \mathrm{R}$ Expression in SHR Elevated CXCL8 and CCL2 expression patterns have been observed in VSMCs and thoracic aortas in SHR. ${ }^{15,17,18)}$ Using immunohistochemical staining, SHR-R was shown to have decreased CXCL8 and CCL2 protein levels, which were similar to the levels observed in WKY-N (Fig. 3A). Furthermore, significant decreases in CXCL8 and CCL2 mRNA expression levels were also detected in the thoracic aortas of SHR-R (Fig. 3B).
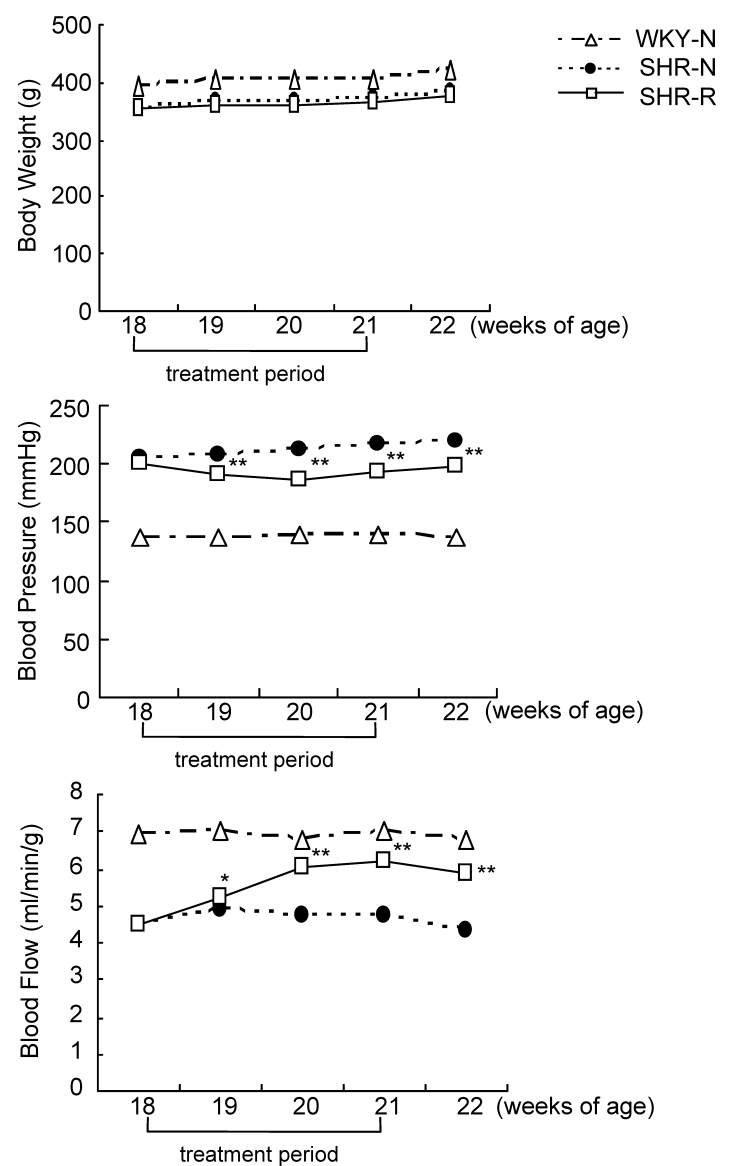

Fig. 1. Reparixin Treatment Decreases Systolic Blood Pressure and Increases Blood Flow in SHR

Body weight, systolic blood pressure and blood flow were measured before, every week during treatment and 1 week after final treatment with reparixin $(5 \mathrm{mg} / \mathrm{kg} / \mathrm{d})$ or normal saline. Systolic blood pressure was measured using the tail-cuff method, and blood flow was measured with a laser blood flowmeter. Results are presented as the mean \pm S.E.M. $(n=5$, each). $* p<0.05$ vs. SHR-N. $* * p<0.01 v s$. SHR-N. WKY-N: WKY treated with normal saline, SHR-N: SHR treated with normal saline, SHR-R: SHR treated with reparixin. 
A
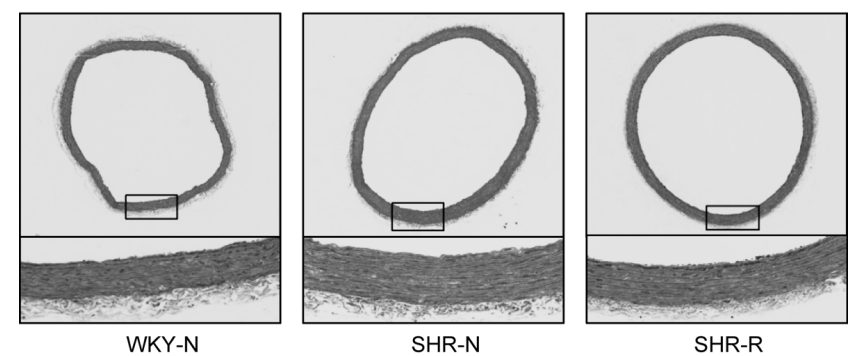

B
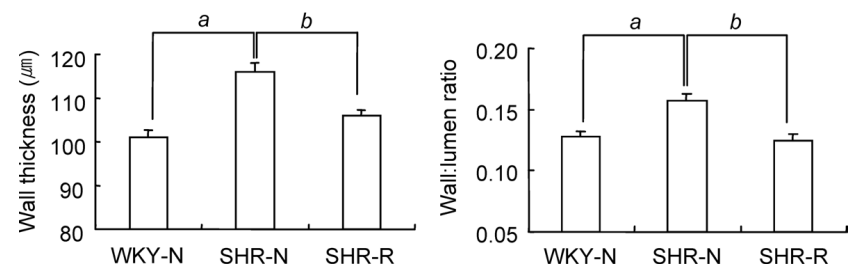

Fig. 2. Reparixin Decreases Thoracic Aorta Wall Thickness in SHR

(A) Aortic cross sections were stained using Masson's trichrome method. Original magnification $\times 40$ (upper) and $\times 200$ (lower) (Zeiss microscope, Oberkochen, Germany). (B) Wall thickness and wall : lumen ratios of the thoracic aorta are shown. Bars represent the mean \pm S.E.M. $\left(n=5\right.$, each). ${ }^{\mathrm{a}} p<0.05 v s$. WKY-N. ${ }^{\mathrm{b}} p<0.05 v s$. SHR-N. WKY-N: WKY treated with normal saline, SHR-N: SHR treated with normal saline, SHR-R: SHR treated with reparixin.

Increased expression of $\mathrm{AT}_{1} \mathrm{R}$ has previously been observed in SHR VSMCs relative to WKY VSMCs. ${ }^{15)}$ Likewise, an increased $\mathrm{AT}_{1} \mathrm{R}$ staining in SHR-N thoracic aortas was observed compared to WKY-N, and reparixin treatment reduced $\mathrm{AT}_{1} \mathrm{R}$ staining and mRNA expression in thoracic aortas (Figs. 4A, B). In addition, decreased $\mathrm{AT}_{2} \mathrm{R}$ staining was observed in the thoracic aortas of SHR-N compared to WKY-N, and reparixin treatment increased $\mathrm{AT}_{2} \mathrm{R}$ protein levels to those found in WKY-N (Fig. 4A). However, $\mathrm{AT}_{2} \mathrm{R}$ mRNA expression was elevated in SHR-N thoracic aortas compared to WKY-N and there was no significant difference in $\mathrm{AT}_{2} \mathrm{R}$ gene expression in SHR-R compared to SHR-N (Fig. 4B). Thus, the in vitro effects of reparixin on Ang II (or CXCL8)-induced Ang II receptors in SHR VSMCs and HUVECs were examined (Fig. 4C). The expression of Ang II (or CXCL8)-induced $\mathrm{AT}_{1} \mathrm{R}$ was decreased by reparixin and the effects of reparixin on Ang II-induced Ang II receptors expression were similar to the effects of reparixin on CXCL8induced Ang II receptors. Reparixin had no significant effect on the expression of Ang II (or CXCL8)-induced $\mathrm{AT}_{2} \mathrm{R}$ expression. In HUVECs, the effects of reparixin on Ang II (or CXCL8)-induced Ang II receptors expression were similar to the effects of reparixin in SHR VSMCs.

Reparixin Decreases 12-LO and ET-1 Expression in SHR SHR have elevated 12-LO and ET-1 expression in VSMCs. ${ }^{19-21)}$ Consistent with these findings, an elevated 12LO and ET-1 expression in the thoracic aortas from SHR-N was observed in this study. Importantly, reparixin treatment led to decreased expression of both mediators (Fig. 5A). In addition, the effect of reparixin on 12-LO and ET-1 expression was examined in SHR VSMCs or HUVECs. In these experiments, the effect of reparixin at concentrations of 0.01 , $0.1,0.5$ and $1 \mu \mathrm{M}$ on CXCL8-treated SHR VSMCs were examined and reparixin was shown to inhibit CXCL8-induced 12-LO and ET-1 expression at concentrations greater than
A

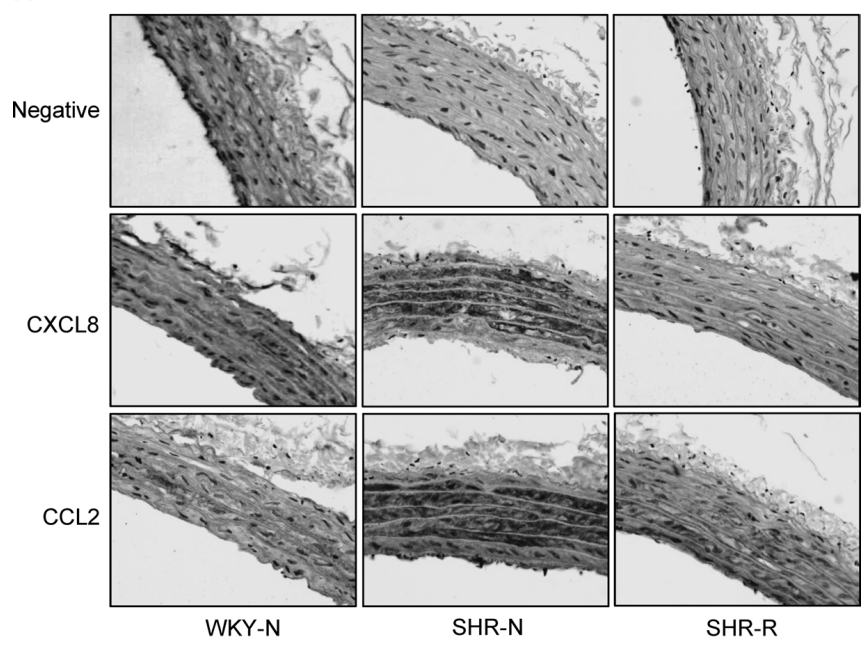

B

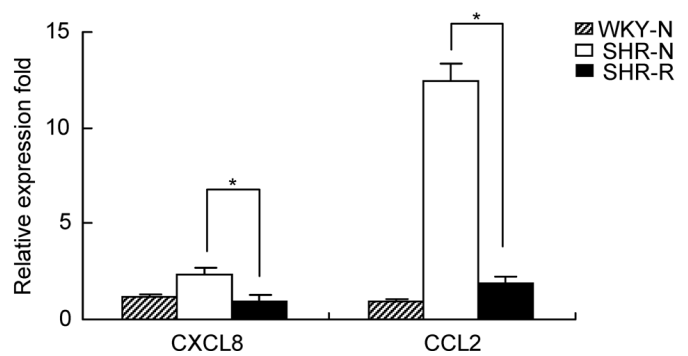

Fig. 3. Reparixin Decreases CXCL8 and CCL2 Expression in SHR Thoracic Aortas

(A) Aortic cross sections were stained immunohistochemically for CXCL8 and CCL2. Original magnification $\times 200$ (Zeiss microscope, Oberkochen, Germany). (B) Total RNA was isolated from the thoracic aortas of each rat group $(n=5$ each $)$, and real-time PCR was performed. Bars represent the mean \pm S.E.M. $* p<0.05 v s$. SHR-N. WKY-N: WKY treated with normal saline, SHR-N: SHR treated with normal saline, SHR-R: SHR treated with reparixin.

$0.01 \mu \mathrm{M}$. In addition, we observed the protein levels by using Western blot, The production of CXCL8-induced 12-LO or ET-1 protein was also decreased by reparixin (Fig. 5B). The inhibitory effect of reparixin $(1 \mu \mathrm{M})$ on CXCL8-induced 12LO and ET-1 mRNA expression was also detected in HUVECs (Fig. 5C).

Next, the effect of losartan, an $\mathrm{AT}_{1} \mathrm{R}$ inhibitor, on the inhibitory action of reparixin on Ang II (or CXCL8)-induced 12-LO and ET-1 expression in SHR VSMCs was examined. The expressions of 12-LO and ET-1 in SHR VSMCs treated with Ang II (or CXCL8) and reparixin together (Ang II/rep, or CXCL8/rep) were decreased by losartan. However, the magnitude of these decreases was similar to the levels observed in SHR VSMCs treated with Ang II (or CXCL8) and losartan, except the expression of ET-1 in SHR VSMCs treated with Ang II/rep and losartan (Figs. 6A, B). The inhibitory action of losartan on Ang II/rep-induced ET-1 expression was greater than the inhibitory effects of losartan on Ang II alone-induced ET-1 expression ( $p<0.05$, Fig. 6B). To confirm this result, we examined the protein levels by using Western blot, The inhibition of Ang II/rep-induced ET-1 protein by losartan was greater than the inhibition of Ang IIinduced ET-1 protein by losartan (Fig. 6C).

Reparixin Increases Plasma NO Levels in SHR and SHR VSMCs The effect of reparixin on NO production 
A
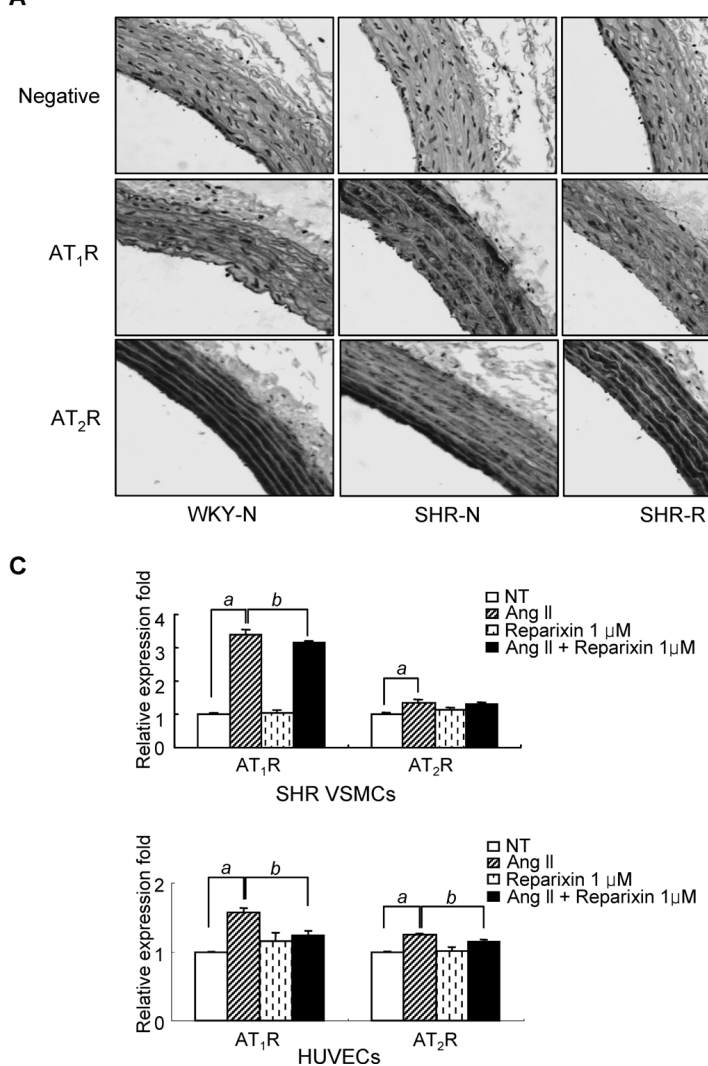

B
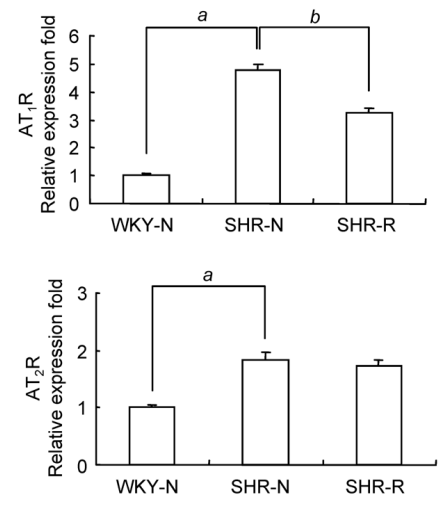

Fig. 4. Reparixin Modulates the Expression of Ang II Receptors in SHR Thoracic Aortas and VSMCs

(A) Thoracic aortas from each rat group were obtained and immunohistochemically labeled for $\mathrm{AT}_{1} \mathrm{R}_{\text {and }} \mathrm{AT} \mathrm{R}_{2} \mathrm{R}$. Original magnification $\times 200$ (Zeiss microscope, Oberkochen, Germany). (B) Total RNA was isolated from thoracic aortas of each rat group ( $n=5$ each), and real time PCR was performed. Bars represent the mean \pm S.E.M. ${ }^{a} p<0.05 v s$. WKYN. ${ }^{b} p<0.05$ vs. SHR-N. WKY-N: WKY treated with normal saline, SHR-N: SHR treated with normal saline, SHR-R: SHR treated with reparixin. (C) SHR VSMCs or HUVECs were pretreated without or with reparixin $(1 \mu \mathrm{M})$ for $30 \mathrm{~min}$. Cells were then treated with or without Ang II (100 nmol/1) or CXCL8 (100 ng/ml) for $2 \mathrm{~h}$. After total RNA was isolated, real-time PCR was performed. Bars represent means \pm S.E.M. from four independent experiments. ${ }^{\mathrm{a}} p<0.05$ vs. untreated VSMCs. ${ }^{\mathrm{b}} p<0.05 v s$. VSMCs treated with Ang II or CXCL8.

was examined in SHR plasma. Plasma NO levels in SHR-N were lower than those in WKY-N (SHR-N: $2.9 \pm 0.1$, WKY$\mathrm{N}: 3.8 \pm 0.2 \mu \mathrm{mol} / 1)$. Reparixin treatment induced a slight increase in plasma NO levels (SHR-R: 3.3 \pm 0.2 , SHR-N: $2.9 \pm 0.1 \mu \mathrm{mol} / 1)$ (Fig. 7A). Consistent with the in vivo results, NO levels in the cell supernatants of untreated SHR VSMCs were lower than those from WKY VSMCs, and NO production was increased slightly in SHR VSMCs treated with reparixin (Fig. 7B).

\section{DISCUSSION}

This study examined the impact of reparixin on blood pressure and hypertension-related mediators in vasculature from SHR.

The SHR and WKY aged 18 weeks, corresponding to the complete established phases of hypertension, were studied. SHR aged less than 5 weeks is known as prehypertensive state, aged 8 to 9 weeks is in developing hypertension, and aged more than 16 weeks is in established hypertension that exhibit severe progressive hypertension. ${ }^{22,23)}$

Blood pressure was shown to be significantly decreased and the expression patterns of hypertension-related vascular mediators were found to be inhibited in SHR-R. Interestingly, the aortic wall thickness was considerably reduced in SHR-R, and we believe that this results in increased blood flow and decreased blood pressure in SHR-R. To further characterize how rat CXCR2 inhibition affects hypertension, $\mathrm{AT}_{1} \mathrm{R}$ and $\mathrm{AT}_{2} \mathrm{R}$ reactivity in reparixin-treated thoracic aortas were examined. The rennin-angiotensin system performs a basic function in regulating blood pressure, and $\mathrm{AT}_{1} \mathrm{R}$ and $\mathrm{AT}_{2} \mathrm{R}$ participate directly in the pathogenesis of hypertension. ${ }^{24,25}$ The $\mathrm{AT}_{1} \mathrm{R}$ mediates most of the pathophysiological effects of Ang II. In this study, inhibition of CXCR2 in SHR led to decreased $\mathrm{AT}_{1} \mathrm{R}$ production and increased $\mathrm{AT}_{2} \mathrm{R}$ production in the thoracic aortas to normal WKY-N levels. AT ${ }_{1} \mathrm{R}$ activation mediates classical Ang II signaling to promote vasoconstriction and VSMC proliferation. In contrast, $\mathrm{AT}_{2} \mathrm{R}$ activation exerts a counter-regulatory effect on $\mathrm{AT}_{1} \mathrm{R}^{24-26)}$ The data presented in this study suggest that reparixin can regulate the production of angiotensin II receptors, which may influence Ang II-induced hypertension. Widdop et al. ${ }^{27)}$ showed that $\mathrm{AT}_{2} \mathrm{R}$ levels are lower in adult SHR mesenteric arteries compared to WKY. The $\mathrm{AT}_{2} \mathrm{R}$ immunohistochemical data obtained in this work suggest that this is also true in the thoracic aorta. However, although we detected the increase of $\mathrm{AT}_{2} \mathrm{R}$ protein in thoracic aorta from SHR-R, we could not detect the increase of $\mathrm{AT}_{2} \mathrm{R}$ mRNA expression in SHR-R thoracic aorta or in Ang II/rep-treated SHR VSMCs. The expression of $\mathrm{AT}_{2} \mathrm{R}$ mRNA was not consistent with the protein level in immunohistochemical data. The expression level of $\mathrm{AT}_{2} \mathrm{R}$ mRNA in SHR-R was almost same to that level in SHR-N. Also, Expression of $\mathrm{AT}_{2} \mathrm{R}$ mRNA was increased in thoracic aortas from SHR-N relative to WKY-N. This in- 
A

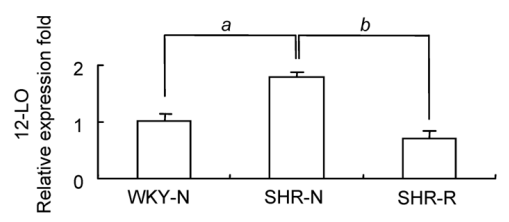

B
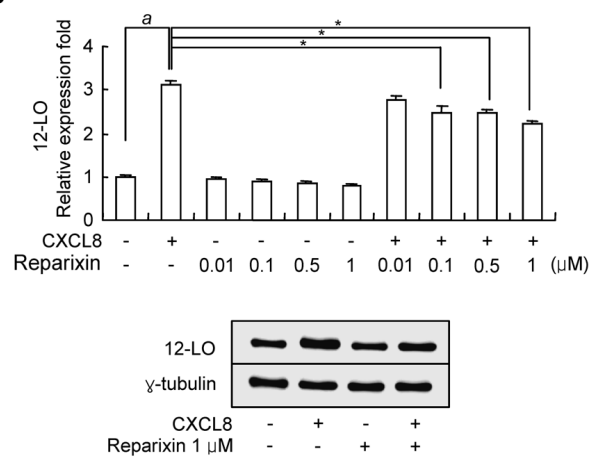
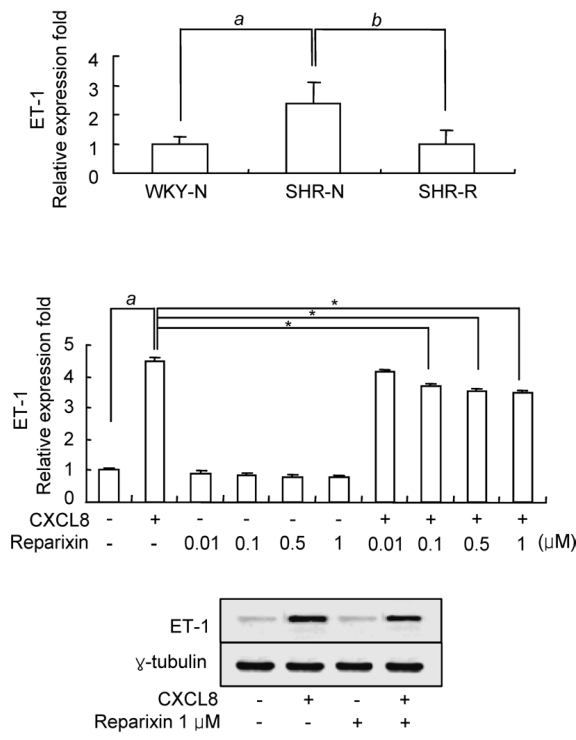

C

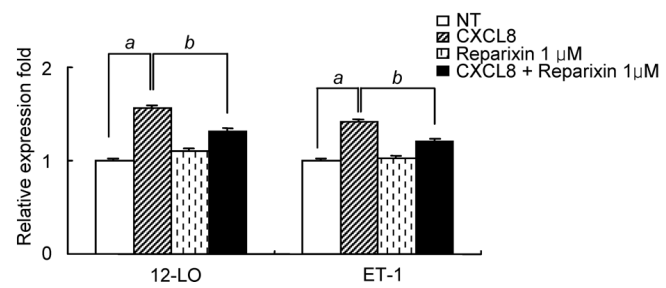

Fig. 5. Treatment of Reparixin Decreases 12-LO and ET-1 Expression in SHR

(A) After total RNA was isolated from thoracic aorta tissues, real-time PCR was performed. Bars represent the mean \pm S.E.M. $\left(n=5\right.$ each). ${ }^{a} p<0.05 v s$. WKY-N. ${ }^{b} p<0.05 v s$. SHR-N. WKY-N: WKY treated with normal saline, SHR-N: SHR treated with normal saline, SHR-R: SHR treated with reparixin. (B) SHR VSMCs were pretreated without or with reparixin $(0.01-1 \mu \mathrm{M}$ or $1 \mu \mathrm{M})$ for $30 \mathrm{~min}$. Cells were then treated with or without CXCL8 $(100 \mathrm{ng} / \mathrm{ml})$ for $2 \mathrm{~h}$. After total RNA and cell lysates were isolated, real-time PCR and immunoblotting were performed. Cell lysates were separated on $10 \%$ SDS-polyacrylamide gels and then immunoblotted with 12 -LO or ET-1 antibody. The data shown are representative of three independent experiments. Bars represent means \pm S.E.M. from four independent experiments. ${ }^{a} p<0.05 v s$. untreated VSMCs. $* p<0.05 v s$. VSMCs treated with CXCL8. (C) HUVECs were pretreated without (NT) or with reparixin $(1 \mu \mathrm{M})$ for $30 \mathrm{~min}$. Cells were then treated with or without CXCL8 (100 ng/ml) for $2 \mathrm{~h}$. After total RNA was isolated, real-time PCR was performed. Bars represent means \pm S.E.M. from three independent experiments. ${ }^{\mathrm{a}} p<0.05$ vs. untreated VSMCs. ${ }^{\mathrm{b}} p<0.05$ vs. VSMCs treated with CXCL8.

crease in $\mathrm{AT}_{2} \mathrm{R}$ mRNA expression is likely a compensatory response to hypertensive vasoconstriction in SHR. De Paolis et al. ${ }^{28)}$ suggested that $\mathrm{AT}_{1} \mathrm{R}$ inhibition may increase $\mathrm{AT}_{2} \mathrm{R}$ promoter activity. In addition, increased $\mathrm{AT}_{2} \mathrm{R}$ expression has also been observed in aortas from SHR treated with an $\mathrm{AT}_{1} \mathrm{R}$ blocker, ${ }^{29)}$ and overexpression of $\mathrm{AT}_{2} \mathrm{R}$ downregulates $\mathrm{AT}_{1} \mathrm{R}$ expression in WKY VSMCs. ${ }^{30)}$ However, although we detected the decrease of $\mathrm{AT}_{1} \mathrm{R}$ and the increase of $\mathrm{AT}_{2} \mathrm{R}$ in thoracic aorta tissues from SHR-R on immunohistochemical data, reparixin has no effect on $\mathrm{AT}_{2} \mathrm{R}$ mRNA expression in thoracic aorta tissues from SHR-R and SHR VSMCs treated with Ang II. This discrepancy between the expression of mRNA and the protein of $\mathrm{AT}_{2} \mathrm{R}$ requires further investigation.

The in vitro inhibitory effect of reparixin on Ang II (or CXCL8)-induced $\mathrm{AT}_{1} \mathrm{R}$ expression was also observed in SHR VSMCs. It is well known that Ang II induces proinflammatory mediators including chemokines. ${ }^{15,26,30,31)}$ The remarkable increase of CXCL8 by Ang II and the increase of $\mathrm{AT}_{1} \mathrm{R}$ by CXCL8 are observed in SHR VSMCs, not in WKY VSMCs. ${ }^{13)}$ Thus, the in vitro inhibitory effect of reparixin on Ang II-induced $\mathrm{AT}_{1} \mathrm{R}$ expression is likely due to the inhibition of Ang II-induced CXCL8 activity by reparixin: namely, reparixin suppresses CXCL8 activation by blocking CXCR2, which in turn suppresses the $\mathrm{AT}_{1} \mathrm{R}$ expression by CXCL8 in

\section{SHR VSMCs.}

Hypertensive experimental rats are hyperresponsive to inflammatory stimuli and chemokine productions. Inflammatory cell infiltration in vascular walls contributes to hypertension-related pathogenesis, and suppression of inflammatory cell infiltration ameliorates hypertension in experimental animal models. ${ }^{18,32-34)}$ Therefore, blocking chemokine production is important for regulating inflammatory reactions in hypertensive vascular walls. CCL2 and its receptor CCR2 have been implicated in hypertensive inflammatory changes in the arterial walls of hypertensive animals. ${ }^{17,31,35)}$ Ishibashi et $a l .{ }^{17)}$ suggested that CCR2 expression in monocytes is critical for vascular inflammation and remodeling in AngII-induced hypertension. CXCL8 is also known to play an important role in monocyte migration into the subendothelial space during the early stages of atherosclerosis. ${ }^{36)}$ Buemi et al. ${ }^{37)}$ suggested that IL-8/CXCL8 directly enhances membrane permeability to $\mathrm{Ca}^{2+}$, thus inducing vasoconstriction in smooth muscle cells during essential hypertension. We previously demonstrated remarkable differences in aortic tissue and VSMC expression of IL-8/CXCL8 in SHR compared to WKY, ${ }^{15)}$ as well as an upregulatory effect of CXCL8 on the production of $12-\mathrm{LO}$ and $\mathrm{AT}_{1} \mathrm{R}$ in SHR VSMCs. ${ }^{13)}$ In this study, treatment with reparixin inhibited expression of CXCL8 and CCL2 in SHR thoracic aortas. These data indi- 

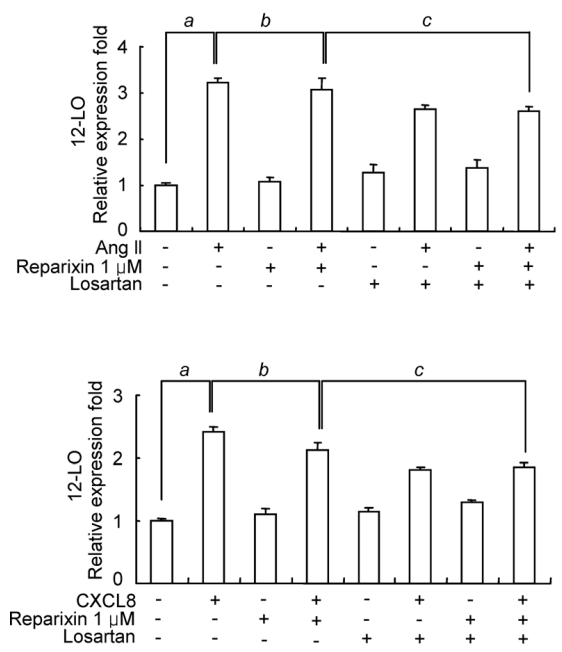

B
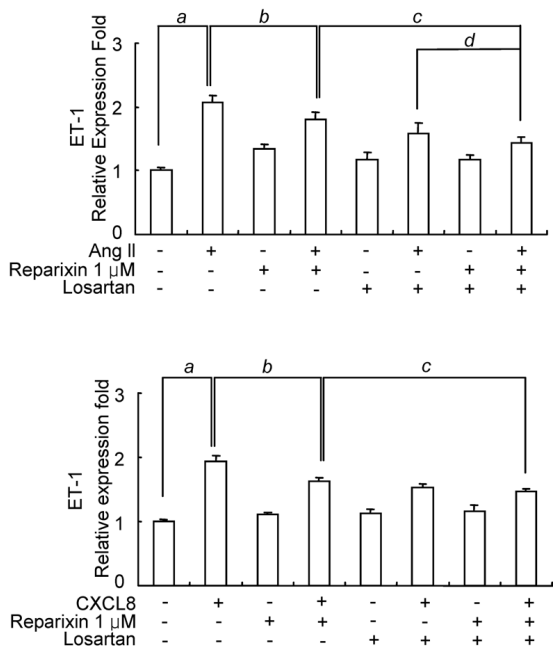

C

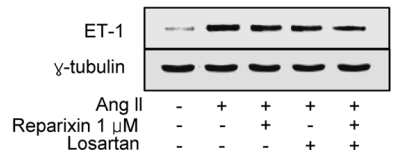

Fig. 6. Losartan Increases the Inhibitory Effect of Reparixin on Ang II-Induced ET-1 mRNA Expression in SHR VSMCs

(A,B) SHR VSMCs were pretreated without or with reparixin $(1 \mu \mathrm{M})$ for $30 \mathrm{~min}$. Cells were then treated with or without Ang II (100 nmol/1) or CXCL8 (100 ng/ml) in the absence or presence of losartan ( $\mathrm{AT}_{1}$ receptor antagonist, $10 \mu \mathrm{mol} / \mathrm{l}$ ) for $2 \mathrm{~h}$. After total RNA was isolated, real-time PCR was performed. Bars represent means \pm S.E.M. from four independent experiments. ${ }^{a} p<0.05 v s$. untreated VSMCs. ${ }^{b} p<0.05$ vs. VSMCs treated with Ang II or CXCL8. ${ }^{c} p<0.05$ vs. SHR VSMCs treated with Ang II and reparixin or CXCL8 and reparixin. ${ }^{\mathrm{d}} p<0.05$ vs. VSMCs treated with Ang II and losartan. (C) SHR VSMCs were pretreated without or with reparixin (1 $\left.\mu \mathrm{M}\right)$ for 30 min. Cells were then treated with or without Ang II $(100 \mathrm{nmol} / \mathrm{l})$ in the absence or presence of losartan $(10 \mu \mathrm{mol} / 1)$ for $2 \mathrm{~h}$. Then, cell lysates were separated on $10 \%$ SDS-polyacrylamide gels and then immunoblotted with ET-1 antibody. The data shown are representative of two independent experiments.

A

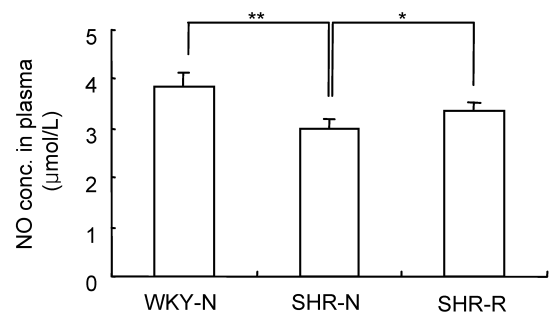

B

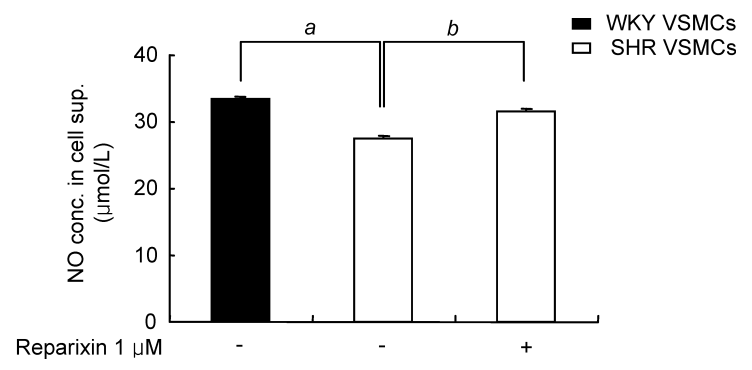

Fig. 7. Reparixin Increases Plasma NO Levels in SHR and VSMCs

(A) Blood samples were obtained via cardiac puncture from each rat group $(n=5$ each). NO levels were approximated by measuring total $\mathrm{NO}_{2}$ and $\mathrm{NO}_{3}$ concentrations using a fluorometric dye. Bars represent the mean \pm S.E.M. $(n=5$ each). $* * p<0.01 v s$. WKY-N. $* p<0.05 v s$. SHR-N. WKY-N: WKY treated with normal saline, SHR-N: SHR treated with normal saline, SHR-R: SHR treated with reparixin. (B) WKY or SHR VSMCs were treated without or with reparixin $(1 \mu \mathrm{M})$ for $4 \mathrm{~h}$. Cell supernatants were then isolated, and NO levels were measured using a fluorometric dye. Bars represent mean \pm S.E.M. from four independent experiments. ${ }^{a} p<0.01 v s$. WKY VSMCs. ${ }^{\mathrm{b}} p<0.01$ vs. untreated SHR VSMCs. cate that reparixin has a downregulatory effect on the production of pro-inflammatory chemokines that are involved in hypertensive inflammatory changes in the arterial walls.

To further characterize the molecular events that lead to decreased blood pressure in SHR-R, the role of reparixin in regulating 12-LO and ET-1 expression was examined. The 12-LO pathway of arachidonic acid metabolism has been linked to cell growth and hypertension pathology. ${ }^{38-40)} \mathrm{Ang}$ II is a potent positive regulator of $12-\mathrm{LO}$ expression and activation in porcine and human VSMCs. ${ }^{41,42)}$ The activities of 12-LO and the 12-LO metabolite 12(S)-hydroxyeicosatetraenoic acid (12(S)-HETE) are increased in SHR, ${ }^{13,20)}$ and a role for 12-LO and 12(S)-HETE has been demonstrated in the pathogenesis of experimental and Ang II-induced hypertension. ${ }^{20,43)}$ Nozawa et al. ${ }^{44)}$ reported that inhibition of the LO pathway lowers blood pressure in renovascular hypertensive rats. Here, reparixin treatment was shown to inhibit 12LO expression in the thoracic aorta walls in SHR. In addition to inhibiting 12-LO expression, reparixin treatment decreased ET-1 expression in the thoracic aortas in SHR. Endothelins increase blood pressure and vascular tone. ${ }^{45}$ Among the three endothelins (ET-1, ET-2 and ET-3), ET-1 is the most abundant and important ET produced by vascular cells. ${ }^{46)}$ ET-1 not only induces vasoconstriction and elicits an inflammatory response by increasing oxidative stress in the vascular walls but it also induces vascular remodeling in experimental hypertensive animals. ${ }^{47}$ ) Therefore, the blood pressure lowering activity of reparixin is thought to be mediated by the inhibition of 12-LO and ET-1 expression. 
In general, the expression of $12-\mathrm{LO}$ and ET-1 is mediated by $\mathrm{AT}_{1} \mathrm{R}$ activation. ${ }^{13,48)}$ In this study, reparixin was shown to inhibit $\mathrm{AT}_{1} \mathrm{R}, 12-\mathrm{LO}$ and $\mathrm{ET}-1$ expression in both the in vivo and in vitro experiments. The expressions of 12-LO and ET-1 in SHR VSMCs treated with Ang II/rep (or CXCL8/rep) and losartan together were decreased compared to those in SHR VSMCs treated with Ang II/rep (or CXCL8/rep) without losartan. In addition, the expression of 12-LO and ET-1 in SHR VSMCs treated with Ang II/rep (or CXCL8/rep) and losartan were lower than those in SHR VSMCs treated with Ang II (or CXCL8) and losartan. Especially, the expression of ET-1 in SHR VSMCs treated with Ang II/rep and losartan was significantly lower than that in SHR VSMCs treated with Ang II and losartan. Thus, downregulation of 12-LO and ET1 expression by reparixin appears to be related to $\mathrm{AT}_{1} \mathrm{R}$ inhibition by reparixin.

Taken together, this study shows that a 3 week blockade of rat CXCR2 activation with reparixin leads to decreased blood pressure in SHR. Reparixin downregulated the hypertensionrelated mediators CCL2, CXCL8, $\mathrm{AT}_{1} \mathrm{R}, 12-\mathrm{LO}, \mathrm{ET}-1$ and NO. These data suggest that activation of CXCR1 and CXCR2 receptor plays an important role in the development of vascular hypertension, possibly by upregulating hypertensive vascular mediators. Importantly, our findings suggest that reparixin might be used therapeutically to treat vascular hypertension.

Acknowledgements This work was supported by a Korean Science and Engineering Foundation (KOSEF) Grant funded by the Korean Government (MEST) (No. 20090063428).

\section{REFERENCES}

1) Bertini R., Allegretti M., Bizzarri C., Moriconi A., Locati M., Zampella G., Cervellera M. N., Di Cioccio V., Cesta M. C., Galliera E., Martinez F. O., Di Bitondo R., Troiani G., Sabbatini V., D’Anniballe G., Anacardio R., Cutrin J. C., Cavalieri B., Mainiero F., Strippoli R., Villa P., Di Girolamo M., Martin F., Gentile M., Santoni A., Corda D., Poli G., Mantovani A., Ghezzi P., Colotta F., Proc. Natl. Acad. Sci. U.S.A., 101, 11791-11796 (2004).

2) Gorio A., Madaschi L., Zadra G., Marfia G., Cavalieri B., Bertini R., Di Giulio A. M., J. Pharmacol. Exp. Ther, 322, 973-981 (2007).

3) Souza D. G., Bertini R., Vieira A. T., Cunha F. Q., Poole S., Allegretti M., Colotta F., Teixeira M. M., Br. J. Pharmacol., 143, 132-142 (2004).

4) Villa P., Triulzi S., Cavalieri B., Di Bitondo R., Bertini R., Barbera S., Bigini P., Mennini T., Gelosa P., Tremoli E., Sironi L., Ghezzi P., Mol. Med., 13, 125-133 (2007).

5) Zarbock A., Allegretti M., Ley K., Br. J. Pharmacol., 155, 357-364 (2008).

6) Howard O. M., Oppenheim J. J., Wang J. M., J. Clin. Immunol., 19, 280-292 (1999).

7) Murphy P. M., Baggiolini M., Charo I. F., Hébert C. A., Horuk R., Matsushima K., Miller L. H., Oppenheim J. J., Power C. A., Pharmacol. Rev., 52, 145-176 (2000).

8) Chuntharapai A., Kim K. J., J. Immunol., 155, 2587-2594 (1995).

9) Dunstan C. A., Salafranca M. N., Adhikari S., Xia Y., Feng L., Harrison J. K., J. Biol. Chem., 271, 32770 -32776 (1996).

10) Casilli F., Bianchini A., Gloaguen I., Biordi L., Alesse E., Festuccia C., Cavalieri B., Strippoli R., Cervellera M. N., Di Bitondo R., Ferretti E., Mainiero F., Bizzarri C., Colotta F., Bertini R., Biochem. Pharmacol., 69, 385-394 (2004).

11) Bizzarri C., Allegretti M., Di Bitondo R., Cervellera M. N., Colotta F., Bertini R., Curr. Med. Chem., 2, 67-79 (2003).

12) Mahida Y. R., Ceska M., Effenberg F., Kurlark L., Lindley I., Hawkey C. J., Clin. Sci., 82, 273-275 (1992).
13) Kim J. H., Kang Y. J., Kim H. S., Immune. Netw., 9, 106-113 (2009).

14) Thomson A. W., Lotze M. T., "The Cytokine Handbook," 4th ed., Vol. II, Academic Press, New York, 2003.

15) Kim H. Y., Kang Y. J., Song I. H., Choi H. C., Kim H. S., Hypertens. Res., 31, 515-523 (2008).

16) Ritt M., Schmieder R. E., Hypertension, 54, 384-387 (2009).

17) Ishibashi M., Hiasa K. I., Zhao Q., Inoue S., Ohtani K., Kitamoto S., Tsuchihasi M., Sugaya T., Charo I. F., Kura S., Tsuzuki T., Ishibashi T., Takeshita A., Egashira K., Circ. Res., 94, 1203-1210 (2004).

18) Capers Q. T., Alexander R. W., Lou P., De Leon H., Wilcox J. N., Ishizaka N., Howard A. B., Taylor W. R., Hypertension, 30, 13971402 (1997).

19) Natarajan R., Rosdahl J., Gonzales N., Bai W., Hypertension, 30, 873-879 (1997).

20) Sasaki M., Hori M. T., Hino T., Golub M. S., Tuck M. L., Am. J. Hypertens., 10, 371-378 (1997).

21) Bae J. J., Kim J. H., Kim H., Kim H. S., J. Bacteriol. Virol., 39, 205216 (2009).

22) Cruzado M. C., Risler N. R., Moatello R. M., Yao G., Schiffrin E. L., Touyz R. M., Am. J. Hypertens., 18, 81-87 (2005).

23) Vaziri N. D., Ni Z., Oveisi F., Hypertension, 31, 1248-1254 (1998).

24) Siragy H. M., Am. J. Kidney Dis., 36, 4-9 (2000).

25) Usui M., Egashira K., Tomita H., Koyanagi M., Katoh M., Shimokawa H., Takeya M., Yoshimura T., Matsushima K., Takeshita A., Circulation, 101, 305-310 (2000).

26) Wolf G., Wenzel U., Burns K. D., Harris R. C., Stahl A. K., Thaiss F., Kidney Int., 61, 1986-1995 (2002).

27) Widdop R. E., Vinh A., Henrion D., Jones E. S., Clin. Exp. Pharmacol. Physiol., 35, 386-391 (2008).

28) De Paolis P., Porcellini A., Giante B., Giliberti R., Lombardi A., Savioia C., Rubattu S., Volpe M., J. Hypertens., 17, 1873-1877 (1999).

29) Cosentino F., Savoia C., Paolis P. D., Francia P., Russo A., Maffei A., Venturelli V., Schiavoni M., Lembo G., Volpe M., Am. J. Hypertens., 18, 493-499 (2005)

30) Jin X. Q., Fukuda N., Su J. Z., Lai Y. M., Suzuki R., Tahira Y., Takagi H., Ikeda I., Kanmatsuse K., Miyazaki H., Hypertension, 39, 1021— 1027 (2002).

31) Chen X. L., Tummala P. E., Olbrych M. T., Alexander R. W., Medford R. M., Circ. Res., 83, 952-959 (1998).

32) Zhang Y., Griendling K. K., Dikalova A., Owens G. K., Talyor W. R., Hypertension, 46, 732-737 (2005).

33) Rodríguez-Iturbe B., Vaziri N. D., Herrera-Acosta J., Johnson R. J., Am. J. Physiol., 286, 606-616 (2004).

34) Dhungana S., Sharrack B., Woodroofe N., Headache, 49, 282-285 (2009).

35) Spinetti G., Wang M., Monticone R., Zhang J., Zhao D., Lakatta E. G., Arterioscler. Thromb. Vasc. Biol., 24, 1397-1402 (2004).

36) Gerszten R. E., Garcia-Zepeda E. A., Lim Y. C., Yoshida M., Ding M. A., Gimbrone M. A., Luster A. D., Luscinskas F. W., Rosenzweig A., Nature (London), 398, 718-723 (1999).

37) Buemi M., Marino D., Floccari F., Ruello A., Nostro L., Aloisi C., Marino M. T., Pasquale G., Corica F., Curr. Med. Res. Opin., 20, 1924 (2004)

38) Preston I. R., Hill N. S., Warburton R. R., Fanburg B. L., Am. J. Lung. Cell. Mol. Physiol., 290, 367-374 (2006).

39) Merched A. J., Ko K., Gotlinger K. H., Serhan C. N., Chan L., FASEB J., 22, 3595-3606 (2008).

40) Reddy M. A., Sahar S., Villeneuve L. M., Lanting L., Natarajan R. Arterioscler. Thromb. Vasc. Biol., 29, 387-393 (2009).

41) Natarajan R., Gu J. L., Rossi J., Gonzales N., Lanting L., Xu L., Nadler J., Proc. Natl. Acad. Sci. U.S.A., 90, 4947-4951 (1993).

42) Kim J. A., Gu J. L., Natarajan R., Rerliner J. A., Nadler J. L., Arterioscler. Thromb. Vasc. Biol., 15, 942-948 (1995).

43) González-Núnez D., Claria J., Rivera F., Roch E., Hypertension, 37, $334-338$ (2001).

44) Nozawa K., Tuck M. L., Golub M., Eggena P., Nadler J. L., Stern N., Am. J. Physiol., 259, H1774-H1780 (1990).

45) Agapitov A. V., Haynes W. G., Jraas., 2, 1-15 (2002).

46) Inoue A., Yanagisawa M., Kimura S., Kasuya Y., Miyauchi T., Goto K., Masaki T., Proc. Natl. Acad. Sci. U.S.A., 86, 2863-2867 (1989).

47) Schiffrin E. L., Vascul. Pharmacol., 43, 19-29 (2005).

48) Dumont Y., D’Amours M., Lebel M., Lariviere R., J. Hypertens., 19, 1479-1487 (2001). 